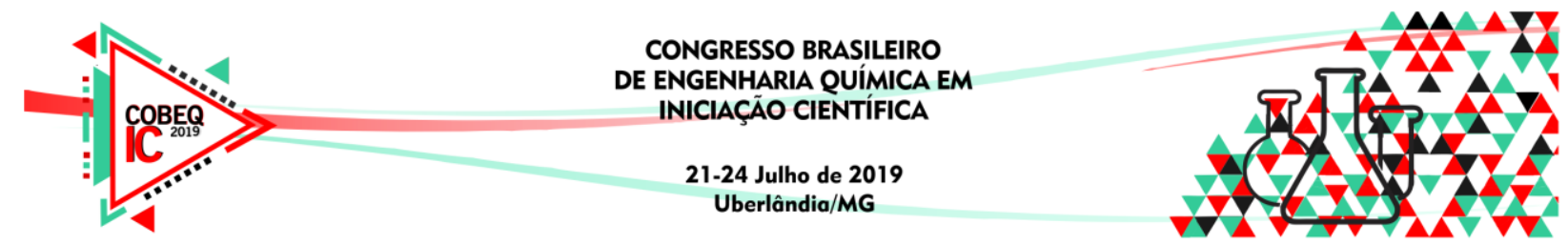

\title{
ESTUDO IN SILICO DO POTENCIAL FARMACOLÓGICO DO ÓLEO ESSENCIAL DOS COMPONENTES MAJORITÁRIOS DO CIPÓ D'ALHO (ADENOCALYMMA ALLIACEUM)
}

\author{
E. MOCHIUTTI ${ }^{1}$, A. L. S. CARVALHO ${ }^{1}$, A. E. S. NASCIMENTO ${ }^{1}$, D. S. B. BRASIL ${ }^{1}$ e M. \\ C. MARTELLI ${ }^{1}$ \\ ${ }^{1}$ Universidade Federal do Pará, Faculdade de Engenharia Química \\ E-mail para contato: mochiuttieric@gmail.com
}

\begin{abstract}
RESUMO - O Cipó d'alho é uma planta encontrada no Brasil, que é utilizada para pela medicina tradicional para diversos tratamentos medicinais. A fim de explorar o potencial farmacológico dos componentes majoritários do óleo essencial dessa planta, utilizou-se métodos computacionais e bancos de dados para predizer o comportamento dessas moléculas. Nesse trabalho foram analisadas as moléculas 3-Vinyl-1,2-dithi-4-ene, Diallyldisulfide, Diallyltrisulfide e Diallyltetrasulfide, obtendo-se resultados de propriedades físico-químicas, farmacocinética, aplicando a regra de Lipinski e predizendo possíveis atividades biológicas dos compostos e comparando com classificações obtidas na literatura.
\end{abstract}

\section{INTRODUÇÃO}

O Cipó d'alho pertence à família Bignoniaceae e é encontrada na região norte do Brasil, com diversas pesquisas que documentam seu uso na medicina tradicional como analgésico, antiartrítico, anti-inflamatório entre outras atividades farmacológicas (SHUKLA et al., 2008). A riqueza da flora brasileira motiva diversos estudos das propriedades dos extratos vegetais e desenvolvimento de produtos baseados em plantas, incluindo produtos farmacêuticos. Contudo, os estudos envolvendo análise de determinados ativos tem elevado custo.

O uso de métodos computacionais é considerado uma ferramenta para o estudo e previsão de propriedades físico-químicas. Umas das técnicas usadas para reduzir custos é a modelagem molecular e a análise de banco de dados, que tem apresentado cada vez mais relevância em diferentes áreas do conhecimento. Atualmente, a indústria farmacêutica é a que mais utiliza-se dessa técnica para prever propriedades e mecanismos de reação. Muitos estudos envolvem a aplicação dessa metodologia para analisar atividade biológica de determinadas substâncias, reduzindo tempo e custo e viabilizando a análise de propriedades que não são possíveis de determinar experimentalmente. (NETO et al., 2010).

Pesquisas realizadas por Zoghbi et al. (2001) identificaram os componentes majoritários do óleo essencial das folhas do Cipó d'alho (Adenocalymma alliaceum) como sendo as substâncias Diallyldisulfide, Diallyltrisulfide, Diallyltetrasulfide e 3-Vinyl-1,2-dithi-4-ene. O 


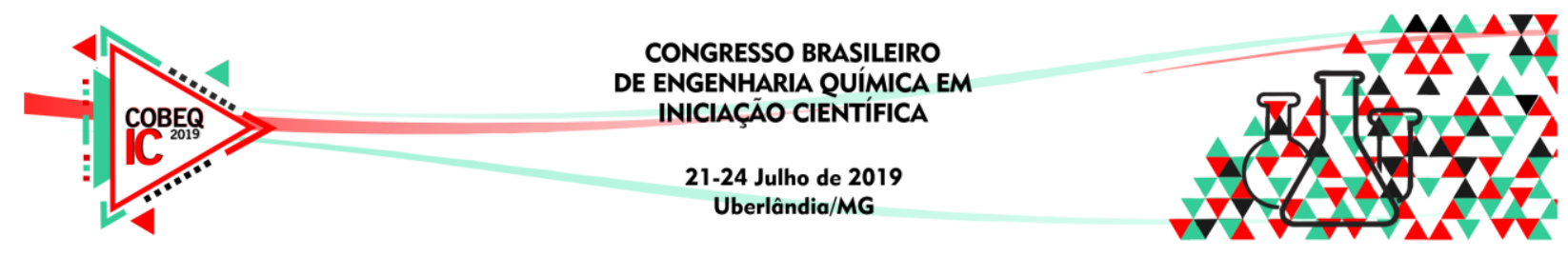

objetivo desse trabalho é, portanto, identificar através de modelagem molecular e banco de dados as propriedades físico-químicas de interesse e farmacológicas de moléculas obtidas a partir do óleo essencial das folhas da Adenocalymma alliaceum.

\section{MATERIAS E MÉTODOS}

Os modelos bidimensionais foram desenhados pelo software MarvinSketch v.17.29 e posteriormente convertidos em modelos tridimensionais usando Avogadro. Os modelos das moléculas identificadas nos trabalhos citados encontram-se na tabela 1 abaixo:

Tabela 1 - Componentes majoritários do óleo essencial das folhas da Adenocalymma alliaceum

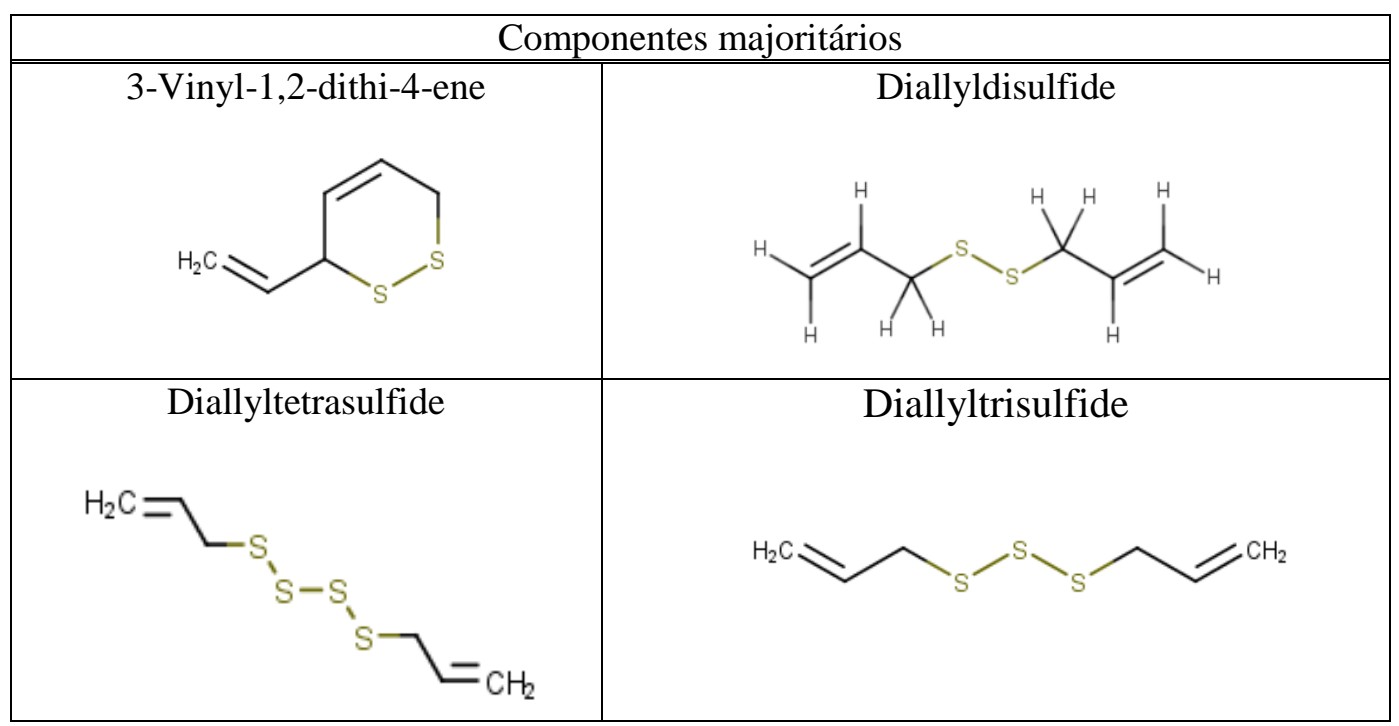

As estruturas foram otimizadas com base no método da teoria do funcional da densidade (DFT) usando a aproximação da densidade funcional híbrida B3LYP, juntamente com a base 6-31G como parâmetros de cálculo pelo programa Gaussian, onde a energia mínima foi verificada. Os cálculos de frequência foram para confirmar os pontos estacionários, garantindo que não houvesse frequências imaginárias, mostrando assim que as estruturas correspondiam à energia local mínima (SOUZA et al., 2018).

Depois de determinar a geometria tridimensional das moléculas, várias propriedades de interesse, incluindo seus descritores eletrônicos, quânticos, físico-químicos e topológicos foram calculados usando o programa Spartan. As propriedades calculadas foram: energia do orbital molecular ocupado mais alto (HOMO), energia do orbital molecular desocupado mais baixo (LUMO) a diferença entre HOMO e LUMO (GAP), massa, área de superfície polar (PSA), partição coeficiente (LogP), doador de ligação de hidrogênio (HBD), aceitador de ligação de hidrogênio (HBA) e com esses resultados foi aplicada a regra de Lipinski. Para a descoberta, a regra de Lipinski prediz que a má absorção é mais provável quando: há mais de 5 doadores de ligações de hidrogênio;10 aceitadores de ligação de hidrogênio; o peso molecular é maior que 500 e; o Log P calculado (LogP) é maior que 5. (LIPINKI et al., 1997) 


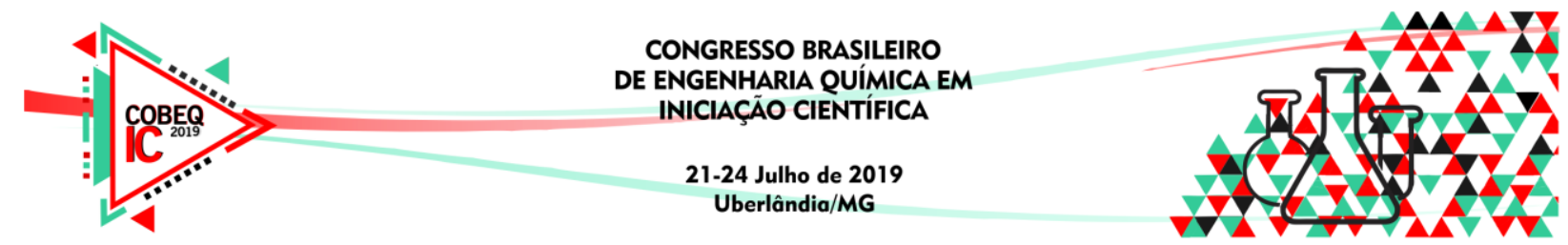

Ferramentas on-line também foram usadas para obter previsões sobre a informação farmacocinética. As seguintes propriedades ADME foram preditas pelo servidor on-line PreADMET: absorção intestinal humana (HIA) e permeabilidade celular Caco-2 in vitro. No mesmo servidor, utilizou-se a ferramenta de predição para a toxicidade das moléculas, verificando, assim, a mutagenicidade (teste de Ames), carcinogenicidade para ratos / camundongos.

O servidor on-line PASS (Prediction of Activity Spectra for Substances), que é baseado em descritores estatísticos foi usado para prever a atividade biológica. Os valores encontrados indicam a probabilidade de o composto estar ativo $(\mathrm{Pa})$ e a probabilidade de estar inativo $(\mathrm{Pi})$, para cada atividade biológica investigada. Para substância ser provavelmente ativa para a atividade biológica ela deve possuir um valor de Pa acima de 0,7 (LAGUNIN et al., 2000), porém, a substância pode ser análoga ao de um agente farmacêutico.

\section{RESULTADOS E DISCUSSÃO}

Na Tabela 2 encontram-se os resultados de energia HOMO, LUMO, GAP, partição coeficiente $(\log \mathrm{P})$, doador de ligação de hidrogênio (HBD), aceitador de ligação de hidrogênio (HBA).

Tabela 2- Propriedades físico-químicas dos componentes majoritários do Cipó d'alho.

\begin{tabular}{|c|c|c|c|c|c|c|c|c|}
\hline Molécula & $\begin{array}{c}\text { HOMO } \\
(\mathrm{eV})\end{array}$ & $\begin{array}{c}\text { LUMO } \\
(\mathrm{eV})\end{array}$ & $\begin{array}{c}\text { GAP } \\
(\mathrm{eV})\end{array}$ & $\begin{array}{c}\text { Massa } \\
(\mathrm{Da})\end{array}$ & $\begin{array}{c}\text { PSA } \\
\left(\mathrm{A}^{2}\right)\end{array}$ & HBD & HBA & LogP \\
\hline \hline 3-Vinyl-1,2-dithi-4-ene & $-5,97$ & $-0,71$ & 5,26 & 144,262 & 0 & 0 & 2 & 2,51 \\
\hline Diallyldisulfide & $-6,4$ & $-0,96$ & 5,44 & 146,278 & 0 & 0 & 2 & 2,95 \\
\hline Diallyltetrasulfide & $-6,43$ & $-1,79$ & 4,64 & 210,410 & 0 & 0 & 4 & 3,95 \\
\hline Diallyltrisulfide & $-6,48$ & $-1,4$ & 5,08 & 178,344 & 0 & 0 & 3 & 3,36 \\
\hline
\end{tabular}

A diferença entre a substância 3-Vinyl-1,2-dithi-4-ene e as outras três é mais evidente devido ao fato de serem estruturalmente diferentes. Em relação as Diallyl di, tri, tetra, pode-se observar que os valores de energia HOMO e LUMO aumentaram de acordo com o número de enxofres na cadeia, sendo a substância Diallyldisulfide com menores valores e a Diallyltetrasulfide. Os resultados de HOMO e LUMO servem de parâmetros para entender a reatividade das moléculas, seu caráter eletro receptor/doador, propriedades óticas e elétricas, e informações sobre a transferência de carga dentro da molécula. O valor de GAP é um indicativo da estabilidade da molécula, baixos valores indicam alta reatividade enquanto valores elevados indicam alta estabilidade (CUNHA et al.,2015). Nos resultados variou de 5,44 eV a 4,64 eV, sendo, portanto, a Diallyltetrasulfide a mais estável e a 3-Vinyl-1,2-dithi4-ene mais instável.

Área de superfície polar molecular (PSA) é um descritor que correlaciona o transporte molecular passivo através de membranas. Para a permeabilidade em células Caco-2 intestinais, os fármacos com um PSA de $60 \AA^{2}$ ou menos, são completamente absorvidos (PAJOUHESH e LENZ, 2005). Portanto, com base nessas classificações, as moléculas do extrato apresentaram permeabilidade celular, com referência aos intervalos descritos. 


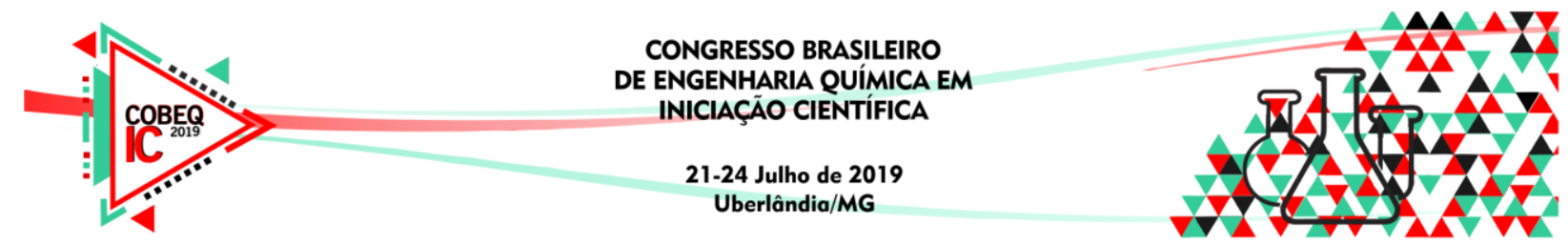

A partir dos valores calculados na Tabela 2, de massa, coeficiente de partição, HBD, HBA e aplicando a regra de Lipinski, obtém-se os resultados de que todas as moléculas se enquadram na regra, mostrando o potencial para a utilização desse extrato para medicamentos.

Na descoberta de novas drogas, a atividade biológica não é o único foco, também é necessário buscar propriedades farmacocinéticas favoráveis e com baixa toxicidade. A predição da absorção (HIA, Pcaco-2), para as moléculas encontram-se na tabela 3.

Tabela 3- Propriedades farmacocinéticas de absorção, distribuição, metabolismo e excreção dos componentes majoritários do Cipó d'alho.

\begin{tabular}{|c|c|c|}
\hline Molécula & Caco2 $(\mathrm{nm} / \mathrm{sec})$ & HIA (\%) \\
\hline \hline 3-Vinyl-1,2-dithi-4-ene & 26.4934 & 98.1691 \\
\hline Diallyldisulfide & 22,0171 & 98,169149 \\
\hline Diallyltetrasulfide & 58.5293 & 99.516 \\
\hline Diallyltrisulfide & 57,6318 & 98,995694 \\
\hline
\end{tabular}

O resultado da absorção intestinal humana é o nível de absorção e biodisponibilidade avaliado a partir da proporção de excreção. Podemos então classificar, baseado no trabalho de Yee (1997), onde os compostos com absorção acima de 70\% são classificados como sendo de alta absorção, portanto, todos os compostos do extrato apresentam alta absorção. O modelo de células Caco-2 tem sido recomendado como um modelo in vitro confiável para a predição da absorção oral de drogas. A classificação de permeabilidade PCaco-2 corresponde a: baixa quando os valores são menores que $4 \mathrm{~nm} / \mathrm{sec}$; média, entre 4 e $70 \mathrm{~nm} / \mathrm{sec}$ e; alta, para valores maiores que $70 \mathrm{~nm} / \mathrm{sec}$. (YAMASHITA et al., 2000), portanto, neste estudo, todos os compostos foram classificados com média permeabilidade.

Na tabela 4 constata-se a toxicidade das moléculas segundo o banco de dados do Preadmet, onde estão os dados do teste de Ames, carcinogenicidade.

Tabela 4-Toxicidade dos componentes majoritários do extrato do Cipó d'alho.

\begin{tabular}{|c|c|c|c|}
\hline Molécula & Mutagenicidade & $\begin{array}{c}\text { Carcinogenicidade } \\
\text { (Ratos) }\end{array}$ & $\begin{array}{c}\text { Carcinogenicidade } \\
\text { (Camundongos) }\end{array}$ \\
\hline \hline 3-Vinyl-1,2-dithi-4-ene & Mutagênico & Não & Não \\
\hline Diallyldisulfide & Mutagênico & Sim & Sim \\
\hline Diallyltetraulfide & Mutagênico & Não & Não \\
\hline Diallyltrisulfide & Mutagênico & Sim & Sim \\
\hline
\end{tabular}

O teste de Ames é um método simples para testar a mutagenicidade de um composto e o teste de carcinogenicidade, normalmente usa ratos ou camundongos, expondo-os a um composto e observa-se a existência de câncer. (CUNHA et al., 2015). Pela tabela de toxicidade pode-se observar que apenas os compostos 3-Vinyl-1,2-dithi-4-ene e Diallyltetraulfide apresentaram resultados negativos para atividade carcinogênica e todas as substâncias apresentaram resultados mutagênicos. 


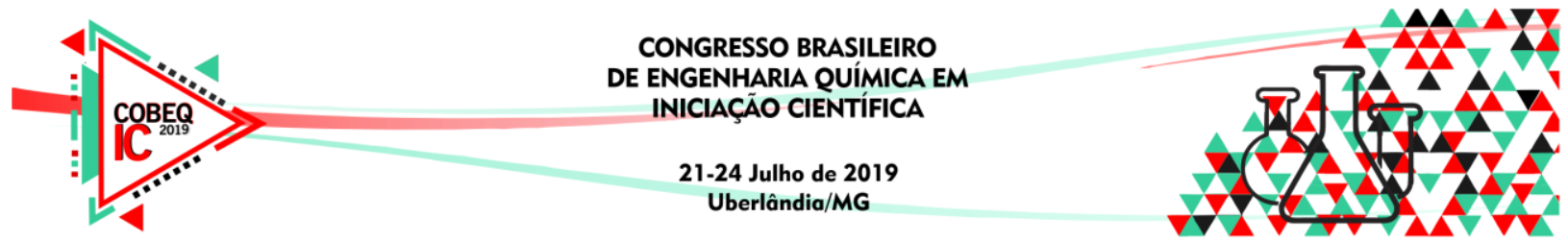

A tabela 5 contém as possíveis atividades biológicas calculadas com valores de probabilidade de ser ativa acima de 0,7 .

Tabela 5- Possíveis atividades biológicas componentes majoritários do Cipó d'alho.

\begin{tabular}{|c|c|c|c|}
\hline Componente & Atividades Biológicas & PA & PI \\
\hline \multirow{3}{*}{ 3-Vinyl-1,2-dithi-4-ene } & Gluconate 2-dehydrogenase (acceptor) inhibitor & 0,901 & 0,004 \\
\hline & Chloride peroxidase inhibitor & 0,832 & 0,003 \\
\hline & Aspulvinone dimethylallyltransferase inhibitor & 0,840 & 0,023 \\
\hline \multirow{3}{*}{ Diallyldisulfide } & Apoptosis agonist & 0,995 & 0,002 \\
\hline & Chemoprotective & 0,950 & 0,000 \\
\hline & Cysteamine dioxygenase inhibitor & 0,943 & 0,001 \\
\hline \multirow{8}{*}{ Diallyltetrasulfide } & Apoptosis agonist & 0,996 & 0,002 \\
\hline & Chemoprotective & 0,959 & 0,000 \\
\hline & Atherosclerosis treatment & 0,941 & 0,003 \\
\hline & Antineoplastic (sarcoma) & 0,921 & 0,001 \\
\hline & Antineoplastic & 0,920 & 0,005 \\
\hline & Antineoplastic (ovarian cancer) & 0,829 & 0,003 \\
\hline & Antineoplastic (cervical cancer) & 0,784 & 0,003 \\
\hline & Prostate cancer treatment & 0,777 & 0,004 \\
\hline \multirow{10}{*}{ Diallyltrisulfide } & Apoptosis agonist & 0,996 & 0,001 \\
\hline & Antineoplastic & 0,986 & 0,004 \\
\hline & Antineoplastic (ovarian cancer) & 0,976 & 0,002 \\
\hline & Antineoplastic (cervical cancer) & 0,970 & 0,002 \\
\hline & Chemoprotective & 0,968 & 0,000 \\
\hline & Antineoplastic (lymphocytic leukemia) & 0,962 & 0,002 \\
\hline & Antineoplastic (sarcoma) & 0,959 & 0,000 \\
\hline & Antineoplastic (non-small cell lung cancer) & 0,935 & 0,002 \\
\hline & Antineoplastic (breast cancer) & 0,925 & 0,003 \\
\hline & Prostate cancer treatment & 0,880 & 0,003 \\
\hline
\end{tabular}

Podendo-se observar que os componentes apresentam atividades biológicas parecidas, devido a semelhança estrutural, com grande destaque para a atividade antineoplásica contra diversos alvos cancerígenos das substâncias Diallyl-di,tri,tetra-sulfide.

\section{CONCLUSÃO}

Com os dados obtidos nesse trabalho é possível identificar as possíveis atividades biológicas antineoplastica presentes nos compostos Diallyl-di,tri,tetra-sulfide e propriedades farmacocinéticas que mostra alta absorção intestinal dos compostos e média permeabilidade celular das moléculas majoritárias isoladas do óleo essencial do Cipó d'alho, porém todos os compostos mostraram ser mutagênicos, sendo que apenas os compostos 3-Vinyl-1,2-dithi-4ene e Diallyltetrasulfide apresentaram resposta negativa para carcinogenicidade, direcionando esses compostos para futuros estudos experimentais para a verificação da viabilidade para a produção de fármacos. 


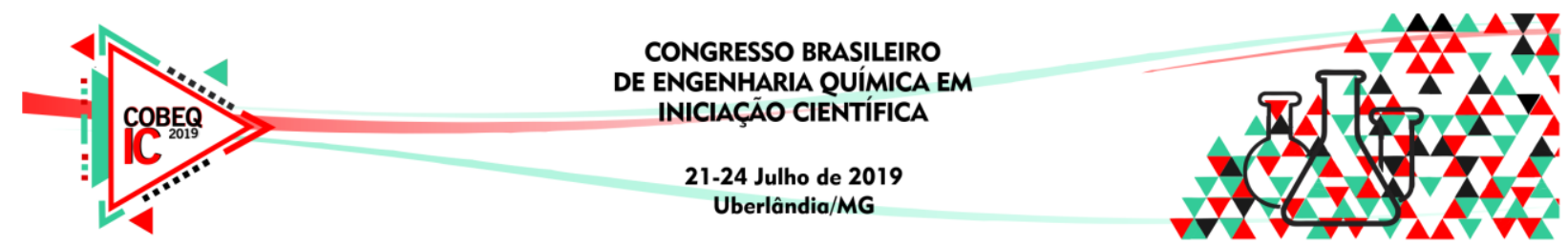

\section{REFERÊNCIAS}

SHUKLA, R.; KUMAR, A.; PRASAD, C. S.; SRIVASTAVA, B.; DUBEY, N. K. Antimycotic and antiaflatoxigenic potency of Adenocalymma alliaceum Miers. on fungi causing biodeterioration of food commodities and raw herbal drugs. International Biodeterioration and Biodegradation, v. 62, n. 4, p. 348-351, 2008.

ZOGHBI, M. D. G. B.; ANDRADE, E. H. A.; MAIA, J. G. S. Volatile constituents from Adenocalymma alliaceum Miers and Petiveria alliacea L., two medicinal herbs of the Amazon. Flavour and Fragrance Journal, v. 17, n. 2, p. 133-135, 2002.

NETO, A. M. J. C.; MOTA, G. V. S.; BORGES, R. S.; ALBUQUERQUE, M. L. S. R-(-)Linalool UV Spectroscopy: The Experimental and Theoretical Study. Journal of Computational and Theoretical Nanoscience, v. 7, n. 2, p. 414-417, 2010.

LIPINSKI, C. A.; LOMBARDO, F.; DOMINY, B. W.; FEENEY, P. J.; Experimental and computational approaches to estimate solubility and permeability in drug discovery and development settings. Adv. Drug Deliv. Rev., v. 23, p. 3-25, 1997

SOUZA, T. N. V.; CARVALHO, S. M. L.; VIEIRA, M. G. A.; SILVA, M. G. C.; BRASIL, D. S. B. Adsorption of basic dyes onto activated carbon: Experimental and theoretical investigation of chemical reactivity of basic dyes using DFT-based descriptors. Applied Surface Science, v. 448, p. 662-670, 2018.

PAJOUHESH, H.; LENZ, G. R. Medicinal chemical properties of successful central nervous system drugs. NeuroRx, v. 2, n. 4, p. 541-553, 2005.

LAGUNIN, A.; STEPANCHIKOVA, A.; FILIMONOV, D.; POROIKOV, V. PASS: Prediction of activity spectra for biologically active substances. Bioinformatics, v. 16, n. 8, p. 747-748, 2000.

YEE, S. In Vitro Permeability Across Caco-2 Cells (Colonic) Can Predict in vivo (Small Intestinal) Absorption in man--Facr or Myth. Pharmaceutical Reaserch, v.14, n. 6, p.763-766, 1997

CUNHA E. L.; SANTOS, C. F.; BRAGA, F. S.; COSTA, J. S.; SILVA, R. C.; FAVACHO, H. A. S.; HAGE-MELIM, L. I. S.; CARVAlHO, J. C. T.; SILVA, C. H. T. P.; SANTOS, C. B. R. Computational investigation of antifungal compounds using molecular modeling and prediction of ADME/tox properties. Journal of Computational and Theoretical Nanoscience, v. 12, n. 10, p. 3682-3691, 2015.

YAMASHITA, S.; Furubayashi, T.; Kataoka, M.; Sakane, T. Optimized conditions for prediction of intestinal drug permeability using Caco-2 cells. European Journal of Pharmaceutical Sciences, v. 10, n. 3, p.195-204, 2000. 